\title{
A Review of the Primary Nutritional and Environmental Factors Associated with Parkinson's Disease
}

\author{
Eleanor Considine ${ }^{1^{*}}$, Lucy Yin ${ }^{2^{*}}$ and Mitra Hartmann ${ }^{3}$ \\ ${ }^{1}$ Skyline High School, Salt Lake City, UT, USA \\ ${ }^{2}$ Aragon High School, San Mateo, CA, USA \\ ${ }^{3}$ Northwestern University, Evanston, IL, USA \\ DOI: https://doi.org/10.47611/jsrhs.v10i2.1482
}

\section{$\underline{\text { ABSTRACT }}$}

Parkinson's disease is a progressive, degenerative nervous system disorder that produces both motor and nonmotor symptoms. This literature review begins by examining evidence for two possible origins for the disease: does it begin in the brain and progress to the gut, or vice versa, or does it begin in both places concurrently? Next, we examine several environmental factors that have been shown to either increase or decrease risk of Parkinson's disease. These are primarily nutritional factors, specifically caffeine, nicotine, and dairy products. Studies in both animals and humans provide weak evidence that increased consumption of low fat dairy is associated with an increased risk of Parkinson's disease development. Additionally, there is strong evidence that nicotine has a neuroprotective effect which also lowers the risk. Finally, there is similarly strong evidence that caffeine exerts neuroprotective effects which lower the overall risk of developing Parkinson's disease.

\section{Introduction: The gut-brain axis: Does PD start in the gut or in the brain or both?}

Traditionally, Parkinson's disease (PD) has been thought to originate in the brain: it is associated with a gradual aggregation of the alpha synuclein protein, which damages nerve cells, in the substantia nigra (Stefanis, 2012).

However, in 2003, neuroanatomist Heiko Braak suggested a possible route by which misfolded alpha-synuclein aggregates travel: the vagus nerve. Cases of idiopathic PD, or a spontaneous form where the origin is uncertain, which comprise the vast majority of PD cases, showed involvement of both the enteric nervous system and the vagus nerve, leading Braak to hypothesize that the aggregates may originate outside of the central nervous system (Braak, Rub, Gai, \& Del Tredici, 2003).

Building off of Braak's observations, researchers injected misfolded alpha synuclein into the intestines of healthy mice. Over the course of 10 months, they observed a buildup of these proteins both in the vagus nerve and the brain. They hypothesized that this neurological disorder may begin in the gut for some patients, indicating that the damage begins in the enteric nervous system, and then travels up to the brain (S. Kim et al., 2019).

Consistent with the possibility that there may be two variants of the disease- one that begins in the brain, and the other in the gut - the symptoms of Parkinson's disease are unique to each patient, some developing intestinal discomfort—such as constipation — first, while others develop neurological symptoms first (Borghammer \& Van Den Berge, 2019). In addition, the progression of symptoms also varies from person to person. The discovery of a gutrelated origin is promising, as it could potentially be easier to prevent Parkinson's disease development if it starts in the gut, knowing that it can be stopped before it enters the brain.

By what possible mechanism(s) could these two putative variants induce Parkinson's disease? As shown in Figure 1 the brain is connected to the gut via the vagus nerve, which offers transportation for proteins. The brain and the gut communicate through a multitude of pathways including the bloodstream, the neuroendocrine system and 
immune response (cytokines) but the vagus nerve offers the most direct form of communication. The vagus nerve is bidirectional which adds to the confusion surrounding the origin of Parkinson's pathology. There have been many studies on the vagus nerve attempting to determine its role in the aggregation of alpha-synuclein in the brain.

A study that used genetic modification to deliberately overproduce alpha synuclein in two groups of mice found that the mice raised in germ free cages had fewer motor deficit symptoms and fewer Lewy Bodies (formed from aggregates of alpha synuclein) in their brain compared to mice raised in a non-sterile environment. In addition, mice raised in the nonsterile environment displayed a decrease in PD symptoms when given an antibiotic, indicating that their microbiome was associated with PD development. Finally, to further support their results, the research team injected gut bacteria from human Parkinson's patients into germ-free mice, and healthy human gut bacteria into others. As expected, the mice injected with the Parkinson's gut microbiota began to display degenerative motor symptoms (Sampson et al., 2016).

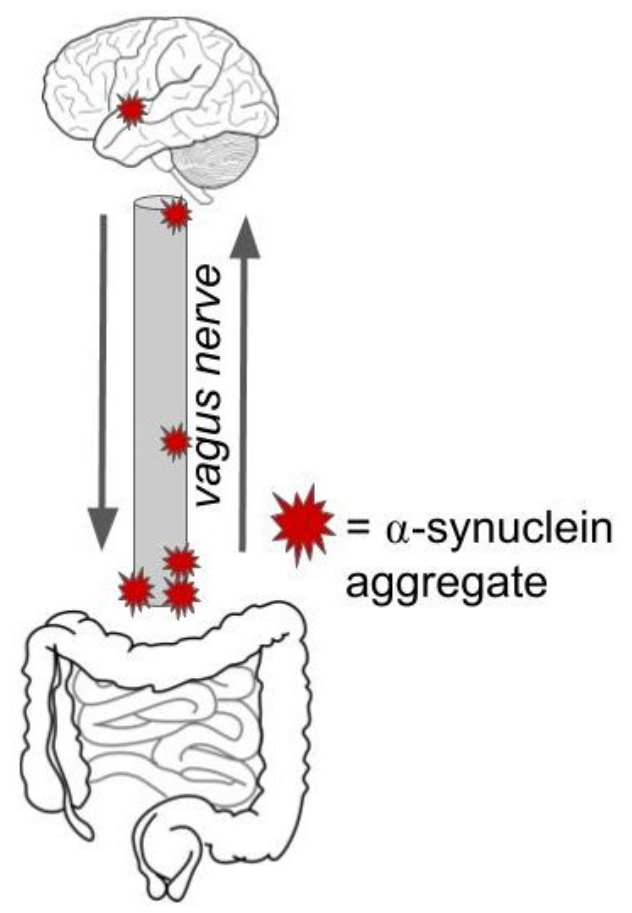

Figure 1. Diagram of the function of the vagus nerve as it is hypothesized in the gut-brain axis argument on Parkinson's pathology. Image of the gut was obtained from https://pixabay.com/vectors/intestines-bowel-guts-intestinal$\underline{293929 /}$ and image of the brain from https://pixy.org/153514/. Both images are CCBY

In addition to rodent studies, evidence from human studies supports the theory of a variant form of PD that originates in the gut. One study found that individuals who underwent truncal vagotomy (a procedure in which both trunks of the vagus nerve are cut) had decreases in both PD symptoms and risks (Svensson et al., 2015).

Other studies of the vagus nerve in humans also provide evidence for a variant that starts in the gut. In one study, researchers studied a cohort of more than 9,000 vagotomized patients. They found that those who underwent a truncal vagotomy were $15 \%$ less likely to develop Parkinson's disease 5 years after their procedure compared to the general population. The truncal vagotomy's association with a decreased rate of developing PD indicates the vagus nerve's possible role as a pathway in which alpha synuclein aggregates travel (Liu, Chan, \& Stoessl, 2017).

If, indeed, at least one type of PD originates in the gut, then this potentially opens new avenues for treatment. Nutrition is one of the most researched aspects of Parkinson's prevention (Boulos, Yaghi, El Hayeck, Heraoui, \& Fakhoury-Sayegh, 2019). To write this review, we performed a broad survey of the literature to reveal five primary 
nutritional and environmental factors associated with risk of developing PD: consumption of dairy, coffee, tea, nicotine, and silymarin. These factors are diagrammed in Figure 2. Although we anticipate that the present review will mostly be relevant to the second mechanism in which it originates in the gut, it is also possible that some factors (e.g., nicotine) are also neuroprotective. When we began this review, we were unaware of the work of Seidl et al. (2014), which reviews research up through 2014 (Seidl, Santiago, Bilyk, \& Potashkin, 2014). The present work represents an independent review of some of the same studies, and also extends the work to include research through 2020.

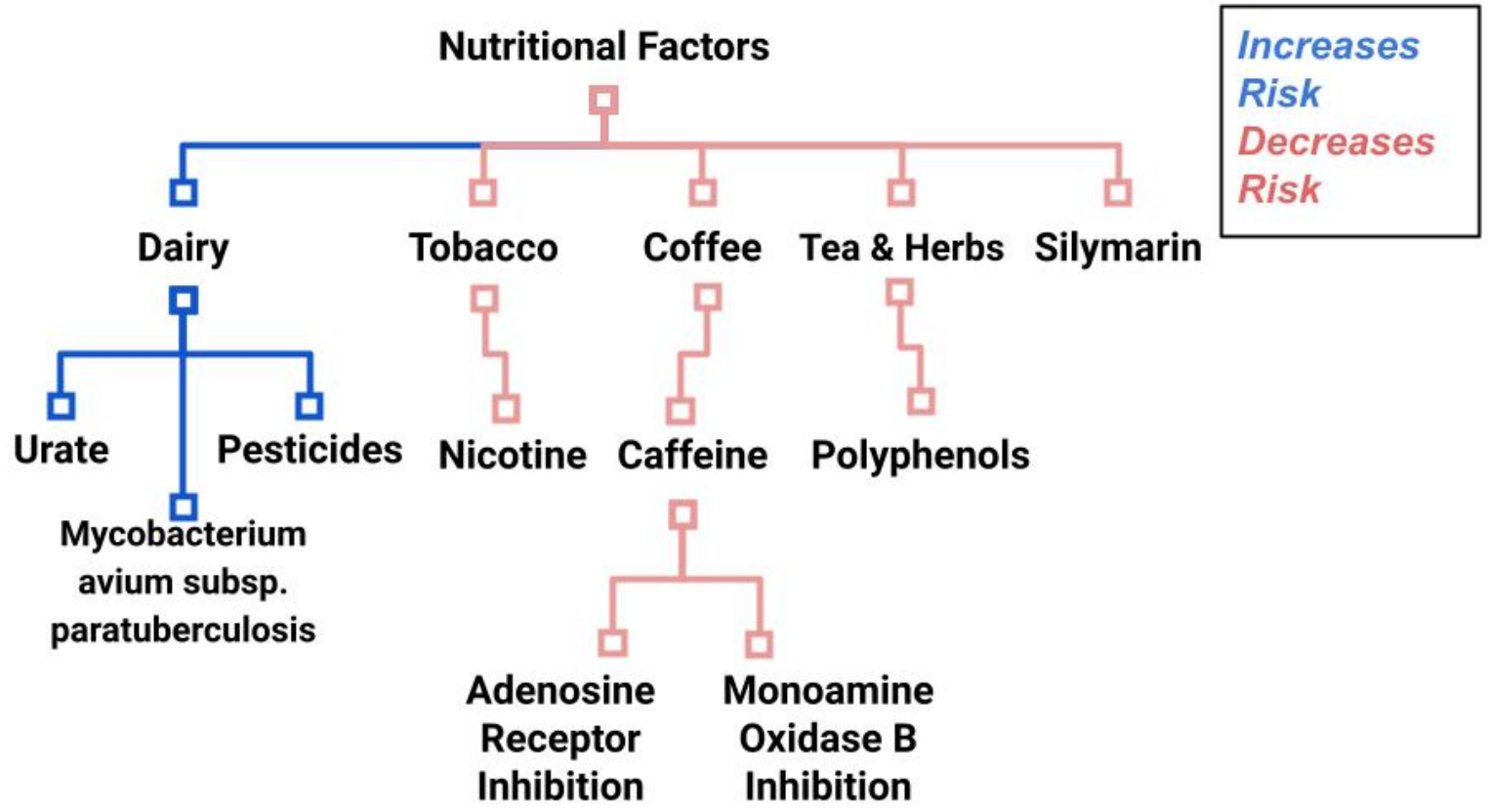

Figure 2. Organizational chart outlining the content of entire review.

\section{Dairy}

Studies in both humans and mice indicate that dairy consumption is associated with an increased risk of the development of Parkinson's disease (Ascherio \& Schwarzschild, 2016; Godos et al., 2020; Hughes et al., 2017; Jiang, Ju, Jiang, \& Zhang, 2014). However, the association is complicated by sex differences as well as the type of dairy product consumed.

One of the largest studies (H. L. Chen et al., 2007) prospectively investigated the association between dairy intake and Parkinson's risk among over 55,000 men and over 70,000 women. These participants were recruited in 1982, and an initial questionnaire was conducted in 1992 on "demographic, medical, environmental, and lifestyle factors and dietary habits." Then, dairy intake was assessed in 1997, 1999, and 2001 through follow-up surveys asking about consumption habits and any new diagnoses, including Parkinson's disease. From 1992 to 2001 , a total of 250 men and 138 women were diagnosed with PD. The authors concluded that dairy consumption was positively associated with increased PD risk for both men and women (H. L. Chen et al., 2007).

Other studies have supported the increased risks of dairy for both men and women, but found that men were at higher risk. One study examined 1,083 PD cases among 304,193 subjects. Overall, the combined risk of PD was found to be $\sim 1.4$ times higher in men than women (Jiang et al., 2014). In terms of types of dairy, the PD risk was 
highest for milk and cheese, and lowest for yogurt and butter. Analysis indicated that PD risk increased by $17 \%$ for every $200 \mathrm{~g} /$ day increment in milk intake, pointing towards a positive correlation.

Yet other studies have found a positive correlation between dairy intake and PD risk for men, but not for women (H. L. Chen, Zhang, Hernan, Willett, \& Ascherio, 2002). This study asked participants how much they consumed of a certain dairy product on a range from "never" to "six or more times a day." These data were then processed into a Food Composition Database at Harvard University, which showed positive associations with Parkinson's disease risk for dairy calcium, dairy vitamin, dairy protein, lactose, and dairy fat intake. However, calcium, vitamin D, and protein from other dietary or supplemental sources were not related to PD risk, leaving lactose and fat. One limitation of this study was that the survey asked subjects to remember their dietary intake; thus, the inconsistency of recalling memories could affect the accuracy of the results (H. L. Chen et al., 2002).

In summary, several epidemiological studies consisting of large samples have consistently found an association between dairy intake - particularly low-fat dairy consumption — and a higher risk for Parkinson's disease (PD). Although the reason for this association is not known, the literature provides three possible reasons: (1) increased consumption of non-fat and low-fat dairy reduces the bioavailability of urate. (2) increased dairy consumption increases exposure to Mycobacterium avium subspecies paratuberculosis (MAP, a bacterium which causes Johne's Disease, an inflammatory bowel disease); (3) increased dairy consumption could be linked to increase in pesticide exposure (Kistner \& Krack, 2014).

\subsection{Dairy -- Potential mechanism: urate levels}

It is known that consumption of low fat and nonfat milk (but not full-fat milk) is associated with a lower concentration of serum urate, an endogenous antioxidant(Jakse, Jakse, Pajek, \& Pajek, 2019). Recent studies suggest that higher concentration of serum urate is associated with a lower risk of PD due to its neuroprotective effect (H. L. Chen \& Marder, 2016; Gao et al., 2008; Kistner \& Krack, 2014).

One study consisted of 4,695 participants that were 55 years and older, who were followed for an average of 9.4 years. Higher serum levels of uric acid were associated with a large decreased risk of PD, which supports the hypothesis that oxidative stress contributes to the risk of PD (de Lau, Koudstaal, Hofman, \& Breteler, 2005). Furthermore, the natural antioxidant and free radical scavenger uric acid seems to have a potential neuroprotective effect (Schlesinger \& Schlesinger, 2008).

Another group examined whether a diet that increases plasma urate level is associated with a reduced risk of Parkinson's disease (Gao et al., 2008). They found that a higher dietary urate index was associated with a lower risk of PD, supporting the suggestion that urate could be a protective factor. A limitation that the authors emphasize is that potential benefits of increased plasma urate levels must be weighed with adverse effects like risk of gout (a form of arthritis caused by too much uric acid in the body).

\subsection{Dairy -- Potential mechanism: Mycobacterium avium s. paratuberculosis (MAP)}

MAP is a bacterium that is known to cause Johne's and Crohn's disease (Dow \& Sechi, 2019). Constant and broad exposure to MAP through dairy products and the environment explains why MAP has been found within granulomas in humans. One group of researchers hypothesizes that PD could originate from MAP exposure. Specifically, the authors hypothesize that, beginning as an enteric infection, MAP initiates a pathologic process that leads to neuroinvasion of the central nervous system via the vagus nerve (similar to a suggested pathway of alpha synuclein from the gut to the brain). They propose that a MAP infection and the resultant PD pathology are similar in that protein quality control systems play a significant role in both (Dow, 2014).

Furthermore, mutations of the LKK2 gene are one of the most common genetic causes of Parkinson's disease (Cabezudo, Baekelandt, \& Lobbestael, 2020). Studies have also indicated that mutations on this gene increase the vulnerability of developing an infection from MAP bacteria (Weindel et al., 2020). While LKK2 gene mutations 
contribute to the development of neurodegenerative diseases, they are also linked to an increased immune response. One study found a link between "neurodegeneration proteins and their first shell interactors in human B lymphocytes" (Nataf, Guillen, \& Pays, 2019). Thus, researchers propose a link between MAP and Parkinson's development, and continue to study associated proteins and genes.

\subsection{Dairy -- Potential mechanism: increased dairy consumption has been linked to increased pes- ticide exposure}

The data are unequivocal that increased pesticide exposure increases risk for Parkinson's (Chade, Kasten, \& Tanner, 2006; Kistner \& Krack, 2014). Two pesticides in particular have been linked: rotenone and paraquat (H. L. Chen \& Marder, 2016).

Evidence for the role of pesticides is found in several studies (Abbott et al., 2016; H. L. Chen \& Marder, 2016). For example, researchers collected milk intake data from 1965 to 1968 in 449 middle aged men. In their postmortem examinations from 1992 to 2004, neuron density was measured in the substantia nigra. Brain residues of heptachlor epoxide, a pesticide found at extreme levels in milk, was also measured. When data were collected, neuron density was lowest in nonsmokers who consumed more than $160 \mathrm{z}$ of milk. Furthermore, residues of the pesticide heptachlor epoxide were found in 9 of 10 brains of milk consuming patients compared to about 6 of 10 in non-dairy consumers. They concluded that milk intake is associated with neuron loss in the substantia nigra and confirmed that pesticides are linked to increased dairy consumption (Abbott et al., 2016).

Another pesticide, dieldrin, has been found in postmortem brain tissue of PD patients, indicating its role in nigral cell death. Although dieldrin has been banned, it continues to contaminate dairy products and meat due to its previous accumulation in the environment (Kanthasamy, Kitazawa, Kanthasamy, \& Anantharam, 2005).

\section{Nicotine}

Because tobacco is notorious for being packed with pesticides (McDaniel, Solomon, \& Malone, 2005), one might think that it would be associated with an increased risk of Parkinson's disease. However, studies consistently show that nicotine seems to offer protection for dopaminergic cells (Ascherio \& Schwarzschild, 2016; Ma et al., 2020). Nicotine has been shown to provide promising therapeutic potential in relation to Parkinson's disease and other neurodegenerative disorders, displaying an inverse relationship between tobacco use and PD development. Nicotine offers neuroprotective effects, specifically in the nigrostriatal pathway (Ascherio \& Schwarzschild, 2016). In non-human primates, doses of nicotine were shown to enhance the effects of levodopa, an amino acid that is used to manage Parkinson's symptoms by increasing dopamine transmission. Nicotine's enhancement of levodopa and its neuroprotective abilities in the CNS make it a strong candidate for treating Parkinson's disease (Quik, O'Leary, \& Tanner, 2008).

Additionally, nicotine has been shown to degrade SIRT6 which is a gene that increases inflammation and promotes the development of PD (Nicholatos et al., 2018). Brain tissue samples and neuronal culture data collected from patients with Parkinson's showed an overall abundance of SIRT6 in the brain, while tobacco users showed decreased levels. Nicotine contributes to a decrease in production of the SIRT6 gene, which could explain its negative correlation with the development of Parkinson's (Nicholatos et al., 2018).

Furthermore, a certain species of Solanaceae, a flowering plant family, are edible and contain nicotine (Nielsen, Franklin, Longstreth, Swanson, \& Checkoway, 2013). Examples include bell peppers, tomatoes, and chilis. In the presence of nicotine, researchers found that dopaminergic neurons were more resistant to the toxic effects of the misfolded proteins, as it was found to reduce the level of mutated and misfolded proteins (Nielsen et al., 2013). 
Surprisingly, a study on Parkinson's patients indicates that nicotine from nicotine patches does not have an effect on the development of Parkinson's disease. For a year, half of the participants received high doses from real nicotine patches, while the other half received placebo patches. At the conclusion of the treatment, the patients were tested on motor, memory, and thinking abilities, as well as behavior and mood through the Unified Parkinson's Disease Rating Scale (UPDRS). UPDRS scores were worse in those treated with the nicotine patch, than those who received a placebo patch. However, the results were not statistically significant. Thus - despite the well-document effect that smoking reduces PD risk - the researchers concluded that nicotine patches at $28 \mathrm{mg}$ per day did not affect the progression of PD (Oertel et al., 2018).

\section{Coffee and Tea}

Repeated consumption of substances with antioxidant properties seems to have neuroprotective effects (Faraone et al., 2019; Kim, Jung, Jeong, \& Chung, 2019). The pathways these substances interact with besides those that attack reactive oxygen species are also involved with protecting against protein aggregation which causes Lewy bodies to form, and the regulation of dopamine in the substantia nigra. All these interactions are potential reasons for why we see a strong correlation between caffeine intake, and simply drinking coffee and/or tea, and a decrease in PD.

Studies are uniform in the finding that caffeine is strongly correlated with a decreased risk of Parkinson's disease (Belvisi et al., 2020; Munoz \& Fujioka, 2018). There are many possible reasons why this correlation is so frequently observed. Here we look at adenosine receptor inhibition, MAO B inhibition, and polyphenols specifically Epigallocatechin gallate (EGCG).

\subsection{Coffee and Tea - Adenosine receptor antagonism}

Caffeine is an adenosine receptor antagonist (Ribeiro \& Sebastiao, 2010). Adenosine is involved in many harmful neuro-inflammatory pathways; thus, its inhibition via caffeine and other antagonists can have general neuroprotective effects (Bobermin, Roppa, \& Quincozes-Santos, 2019). Studies have shown that caffeine's neuroprotective effects at least partially emerge from regulation of adenosine receptors.

In a study on $C$. elegans caffeine once again was shown to protect dopaminergic neurons from neurodegeneration in a dopamine rich environment through the regulation of adenosine receptors (Manalo \& Medina, 2018). Similar results were found in rotenone-induced PD mice where caffeine components through antioxidant properties prevented neurodegeneration (Miyazaki et al., 2018). In MPTP induced rodents and non-human primates, caffeine was found to protect dopaminergic neurons in the substantia nigra through regulation of striatum specific adenosine receptor A(2A)R (Munoz \& Fujioka, 2018). In species from worms to monkeys, adenosine receptor antagonists have been shown to reduce inflammation and increase the number of surviving dopaminergic neurons in the brain.

Because the beneficial effect of caffeine through its interaction with adenosine receptors is so clear, adenosine antagonists have been found to be an effective therapy in PD patients. It has been suggested that adenosine receptor antagonists would be most helpful in treating non-motor symptoms, and decreasing negative side effects of levodopa - the leading Parkinson's medication (Domenici et al., 2019). Adenosine receptor antagonists have been approved by the FDA and used successfully in patients with PD in Japan when administered in conjunction with levodopa (J. F. Chen \& Cunha, 2020).

One group created a model outlining the structures involved in the interaction between adenosine antagonists and dopamine in striatopallidal neurons (A2AR-D2R heterotetramer-AC5 complex), which supported potential treatments using adenosine receptor antagonists (Ferre et al., 2018). 


\subsection{Coffee and Tea - Monoamine oxidase inhibition}

Another possible process by which caffeine protects against Parkinson's is through monoamine oxidase Type B (MAO). MAO is an enzyme that breaks down neurotransmitters such as dopamine and acetylcholine (Dhiman, Malik, \& Khatkar, 2018). Introducing MAO inhibitors causes increases in the amount of dopamine in the brain, which improves the motor symptoms associated with PD (Cesura, 2007). Coffee is a weak MAO inhibitor on its own (Herraiz \& Chaparro, 2006) but some studies have tested caffeine's effect in combination with other MAO inhibitors.

There have been experiments in which a MAO inhibitors in combination with existing Parkinsonian combatant substances (including caffeine) yields "increased extracellular dopamine levels in the striatum" in rats (Faro, Justo, Alfonso, \& Duran, 2020). Further rat studies showed similar results as far as a combination of MAO inhibition and caffeine resulting in neuroprotective effects (Kasabova-Angelova et al., 2020). In a similar study, one caffeine derivative compound (out of eight tested) was found to have neuroprotective effects in terms of enzyme activity and viability (Kondeva-Burdina, Georgieva, Kasabova-Angelova, Tzankova, \& Zlatkov, 2019).

Finally, we have looked at the combination of adenosine receptor antagonists and MAOB inhibitors. The use of dual-acting ligands targeting both sites induced neuroprotective effects (Kuder et al., 2020). Some studies looked at adenosine receptor antagonists' effect on motor symptoms. One group found it helped ease gait freezing in human subjects (Iijima et al., 2019). Another group found a decrease in tremors and catalepsy using a light-activated antagonist in mice (Taura et al., 2018).

\subsection{Coffee and Tea - Polyphenols}

Polyphenols, especially those found in tea, have been associated with a decreased risk of PD as well. Green tea catechins, a type of polyphenol, have been found to inhibit microglial activation and thus reduce inflammation (Farkhondeh et al., 2020). Other groups have identified many neuroprotective targets that catechins interact with, and results have been positive in some in vitro and animal models but have not yet been proven effective for prevention in humans (Farzaei, Bahramsoltani, Abbasabadi, Braidy, \& Nabavi, 2019). Polyphenol catechins protect against glutamate-induced oxidative stress via cell apoptosis proteins and other related enzymes (He, Xu, Yang, \& Sun, 2019). Along with a general decrease in inflammatory activity, one study investigated polyphenol's effect on misfolded protein aggregation, another known risk factor of PD (Yan et al., 2018).

Epigallocatechin gallate, or EGCG, the green tea polyphenol, is a major component of this discussion. This type of catechin with antioxidant properties works to prevent the buildup of protein fibers. This means that when used preventatively, EGCG could combat alpha synuclein aggregation (Pervin et al., 2018). In addition to its neuroprotective effects, EGCG has been shown to decrease motor symptoms in rotenone-induced PD mice (Tseng et al., 2020). The effect of green tea catechins has been statistically investigated with the conclusion that there is a significant correlation between consumption of non-black tea and decreased risk of PD (Zhen et al., 2019). Additionally, EGCG has been seen to interact with gut microbiota on some level. In one study, administration of EGCG resulted in major compositional changes of gut microbiota. Furthermore, the beneficial effects that the EGCG treatment brought about were stopped with the introduction of antibiotics and blocking genes related to the microbiota (Xu et al., 2020).

In the context of treatment, EGCG has also proven helpful. Through its interaction with the immune response, EGCG protected substantia nigra pars compacta cells from toxicity and restored some motor function in MPTPinduced PD mice (Zhou, Zhu et al. 2018). Similar results were found in 6-OHDA induced PD rats, in that EGCG treatment decreased a-synuclein expression and apoptosis, and improved motor function (Zhou, Chen, $\mathrm{Hu}, \mathrm{CaO}, \&$ Yang, 2019). EGCG and other tea polyphenols have illuminated many possible drug targets.

Overall, polyphenols and specifically catechins, interact with antioxidant pathways, gut microbiota, pathways related to motor symptoms of PD and result in neuroprotective effects. 


\section{Silymarin}

Silymarin is an extract of the milk thistle seeds normally used to treat liver disorders, but has been discovered to be a neuroprotective agent against neurologic diseases like Parkinson's disease (Baluchnejadmojarad, Roghani, \& Mafakheri, 2010).

One group investigated the antioxidant effects of silymarin as well as its involvement with $\mathrm{Na}+/ \mathrm{K}+$-ATPase and monoamine oxidase activity in a rat brain. Silymarin was chosen because it displayed a total antioxidant capacity. In vitro data from the study suggests that silymarin's antioxidant and modulatory effects on MAO and $\mathrm{Na}+/ \mathrm{K}+-$ ATPase activity offer neuroprotective effects (de Oliveira et al., 2015).

Neuroprotective effects including various "antioxidant mechanisms, kinases involved in cell signaling pathways, inhibition of the inflammatory response generated during neurodegeneration, neurotrophic effects, regulation of neurotransmitters and inhibition of apoptosis" are in effect when silymarin is present. However, the bioavailability of silymarin is low, and the neuroprotective efficacy still needs to be tested further in humans (Devi, Malar, Braidy, Nabavi, \& Nabavi, 2017).

\section{Conclusion}

Currently, we have come across substantial evidence that supports that nicotine, caffeine, and silymarin have a neuroprotective effect that decreases risk of developing Parkinson's disease. In contrast, we found weaker conclusions that indicate that low-fat dairy is associated with an increased risk.

Overall, our review has left us with more questions than answers, and we list some potential directions for future research below.

There is clearly a difference between the sexes when it comes to prevention and development of Parkinson's in humans, but we see no mention of it in rodent studies. Is it significant only in human patients? If not, further experimentation on rodents with a focus on sex dependency may reveal helpful information for treatment methods.

We also see very few studies on drug-free patients. Is there an interaction between levodopa or other common PD drugs and gut microbiota? Also, how do these mechanisms for neuroprotection change for patients who are taking medication and those who are not?

Studies have found that nicotine patches do not provide the same neuroprotective effect as when nicotine is consumed through tobacco. Why doesn't transdermal nicotine have a neuroprotective effect?

We lack definitive answers of why low-fat dairy is associated with an increased risk of developing PD. Information about the chemical breakdown of dairy or its relationship with the gut microbiota would be helpful. We also wonder why low-fat dairy is associated with an increased risk, but high-fat dairy is not.

\section{Acknowledgments}

We would like to thank our families for support, encouragement, and reading a draft of the manuscript. This work was partially sponsored by award NSF-IOS 1558068 to MH.

\section{References}

Abbott, R. D., Ross, G. W., Petrovitch, H., Masaki, K. H., Launer, L. J., Nelson, J. S., . . . Tanner, C. M. (2016). Midlife milk consumption and substantia nigra neuron density at death. Neurology, 86(6), 512-519. doi:10.1212/wnl.0000000000002254 
Ascherio, A., \& Schwarzschild, M. A. (2016). The epidemiology of Parkinson's disease: risk factors and prevention. Lancet Neurology, 15(12), 1255-1270. Retrieved from < Go to ISI>://WOS:000386315700021

Baluchnejadmojarad, T., Roghani, M., \& Mafakheri, M. (2010). Neuroprotective effect of silymarin in 6hydroxydopamine hemi-parkinsonian rat: Involvement of estrogen receptors and oxidative stress. Neuroscience Letters, 480(3), 206-210. doi:10.1016/j.neulet.2010.06.038

Belvisi, D., Pellicciari, R., Fabbrini, G., Tinazzi, M., Berardelli, A., \& Defazio, G. (2020). Modifiable risk and protective factors in disease development, progression and clinical subtypes of Parkinson's disease: What do prospective studies suggest? Neurobiology of Disease, 134. doi:10.1016/j.nbd.2019.104671

Bobermin, L. D., Roppa, R. H. A., \& Quincozes-Santos, A. (2019). Adenosine receptors as a new target for resveratrol-mediated glioprotection. Biochimica Et Biophysica Acta-Molecular Basis of Disease, 1865(3), 634-647. doi:10.1016/j.bbadis.2019.01.004

Borghammer, P., \& Van Den Berge, N. (2019). Brain-First versus Gut-First Parkinson's Disease: A Hypothesis. Journal of Parkinsons Disease, 9, S281-S295. doi:10.3233/jpd-191721

Boulos, C., Yaghi, N., El Hayeck, R., Heraoui, G., \& Fakhoury-Sayegh, N. (2019). Nutritional Risk Factors, Microbiota and Parkinson's Disease: What Is the Current Evidence? Nutrients, 11(8). doi: $10.3390 /$ nu11081896

Braak, H., Rub, U., Gai, W. P., \& Del Tredici, K. (2003). Idiopathic Parkinson's disease: possible routes by which vulnerable neuronal types may be subject to neuroinvasion by an unknown pathogen. Journal of Neural Transmission, 110(5), 517-536. doi:10.1007/s00702-002-0808-2

Cabezudo, D., Baekelandt, V., \& Lobbestael, E. (2020). Multiple-Hit Hypothesis in Parkinson's Disease: LRRK2 and Inflammation. Frontiers in Neuroscience, 14, 8. doi:10.3389/fnins.2020.00376

Chade, A. R., Kasten, M., \& Tanner, C. M. (2006). Nongenetic causes of Parkinson's disease. Journal of Neural Transmission-Supplement(70), 147-151. Retrieved from <Go to ISI >://WOS:000240329000024

Chen, H. L., \& Marder, K. (2016). Milk consumption and the risk of nigral degeneration. Neurology, 86(6), 496497. doi:10.1212/wnl.0000000000002268

Chen, H. L., O'Reilly, E., McCullough, M. L., Rodriguez, C., Schwarzschild, M. A., Calle, E. E., . . Ascherio, A. (2007). Consumption of dairy products and risk of Parkinson's disease. American Journal of Epidemiology, 165(9), 998-1006. doi:10.1093/aje/kwk089

Chen, H. L., Zhang, S. M. M., Hernan, M. A., Willett, W. C., \& Ascherio, A. (2002). Diet and Parkinson's disease: A potential role of dairy products in men. Annals of Neurology, 52(6), 793-801. doi:10.1002/ana.10381

Chen, J. F., \& Cunha, R. A. (2020). The belated US FDA approval of the adenosine A(2A) receptor antagonist istradefylline for treatment of Parkinson's disease. Purinergic Signalling, 16(2), 167-174. doi:10.1007/s11302-020-09694-2 
de Lau, L. M. L., Koudstaal, P. J., Hofman, A., \& Breteler, M. M. B. (2005). Serum uric acid levels and the risk of Parkinson disease. Annals of Neurology, 58(5), 797-800. doi:10.1002/ana.20663

de Oliveira, D. R., Schaffer, L. F., Busanello, A., Barbosa, C. P., Peroza, L. R., de Freitas, C. M., . . Fachinetto, R. (2015). Silymarin has antioxidant potential and changes the activity of $\mathrm{Na}+\mathrm{K}+$-ATPase and monoamine oxidase in vitro. Industrial Crops and Products, 70, 347-355. doi:10.1016/j.indcrop.2015.03.060

Devi, K. P., Malar, D. S., Braidy, N., Nabavi, S. M., \& Nabavi, S. F. (2017). A Mini Review on the Chemistry and Neuroprotective Effects of Silymarin. Current Drug Targets, 18(13), 1529-1536. doi:10.2174/1389450117666161227125121

Dhiman, P., Malik, N., \& Khatkar, A. (2018). 3D-QSAR and in-silico Studies of Natural Products and Related Derivatives as Monoamine Oxidase Inhibitors. Current Neuropharmacology, 16(6), 881-900. doi:10.2174/1570159x15666171128143650

Domenici, M. R., Ferrante, A., Martire, A., Chiodi, V., Pepponi, R., Tebano, M. T., \& Popoli, P. (2019). Adenosine $\mathrm{A}(2 \mathrm{~A})$ receptor as potential therapeutic target in neuropsychiatric disorders. Pharmacological Research, 147. doi:10.1016/j.phrs.2019.104338

Dow, C. T. (2014). M. paratuberculosis and Parkinson's disease - Is this a trigger. Medical Hypotheses, 83(6), 709712. doi:10.1016/j.mehy.2014.09.025

Dow, C. T., \& Sechi, L. A. (2019). Cows Get Crohn's Disease and They're Giving Us Diabetes. Microorganisms, 7(10). doi:10.3390/microorganisms7100466

Faraone, I., Rai, D. K., Russo, D., Chiummiento, L., Fernandez, E., Choudhary, A., \& Milella, L. (2019). Antioxidant, Antidiabetic, and Anticholinesterase Activities and Phytochemical Profile of Azorella glabra Wedd. Plants-Basel, 8(8). doi:10.3390/plants8080265

Farkhondeh, T., Pourbagher-Shahri, A. M., Ashrafizadeh, M., Folgado, S. L., Rajabpour-Sanati, A., Khazdair, M. R., \& Samarghandian, S. (2020). Green tea catechins inhibit microglial activation which prevents the development of neurological disorders. Neural Regeneration Research, 15(10), 1792-1798. doi:10.4103/1673-5374.280300

Faro, L. R. F., Justo, L. A., Alfonso, M., \& Duran, R. (2020). Possible synergies between isatin, an endogenous MAO inhibitor, and antiparkinsonian agents on the dopamine release from striatum of freely moving rats. Neuropharmacology, 171. doi:10.1016/j.neuropharm.2020.108083

Farzaei, M. H., Bahramsoltani, R., Abbasabadi, Z., Braidy, N., \& Nabavi, S. M. (2019). Role of green tea catechins in prevention of age-related cognitive decline: Pharmacological targets and clinical perspective. Journal of Cellular Physiology, 234(3), 2447-2459. doi:10.1002/jcp.27289

Ferre, S., Bonaventura, J., Zhu, W., Hatcher-Solis, C., Taura, J., Quiroz, C., . . Zwilling, D. (2018). Essential Control of the Function of the Striatopallidal Neuron by Pre-coupled Complexes of Adenosine A(2A)Dopamine D-2 Receptor Heterotetramers and Adenylyl Cyclase. Frontiers in Pharmacology, 9. doi:10.3389/fphar.2018.00243 
Gao, X., Chen, H. L., Choi, H. K., Curhan, G., Schwarzschild, M. A., \& Ascherio, A. (2008). Diet, urate, and Parkinson's disease risk in men. American Journal of Epidemiology, 167(7), 831-838. doi:10.1093/aje/kwm385

Godos, J., Tieri, M., Ghelfi, F., Titta, L., Marventano, S., Lafranconi, A., . . Grosso, G. (2020). Dairy foods and health: an umbrella review of observational studies. International Journal of Food Sciences and Nutrition, 71(2), 138-151. doi:10.1080/09637486.2019.1625035

He, J. T., Xu, L., Yang, L., \& Sun, C. X. (2019). Anti-oxidative effects of catechins and theaflavins on glutamateinduced HT22 cell damage. Rsc Advances, 9(37), 21418-21428. doi:10.1039/c9ra02721a

Herraiz, T., \& Chaparro, C. (2006). Human monoamine oxidase enzyme inhibition by coffee and beta-carbolines norharman and harman isolated from coffee. Life Sciences, 78(8), 795-802. doi:10.1016/j.1fs.2005.05.074

Hughes, K. C., Gao, X., Kim, I. Y., Wang, M. L., Weisskopf, M. G., Schwarzschild, M. A., \& Ascherio, A. (2017). Intake of dairy foods and risk of Parkinson disease. Neurology, 89(1), 46-52. doi:10.1212/wnl.0000000000004057

Iijima, M., Orimo, S., Terashi, H., Suzuki, M., Hayashi, A., Shimura, H., . . Okuma, Y. (2019). Efficacy of istradefylline for gait disorders with freezing of gait in Parkinson's disease: A single-arm, open-label, prospective, multicenter study. Expert Opinion on Pharmacotherapy, 20(11), 1405-1411. doi:10.1080/14656566.2019.1614167

Jakse, B., Jakse, B., Pajek, M., \& Pajek, J. (2019). Uric Acid and Plant-Based Nutrition. Nutrients, $11(8), 15$. doi:10.3390/nu11081736

Jiang, W. J., Ju, C. X., Jiang, H., \& Zhang, D. F. (2014). Dairy foods intake and risk of Parkinson's disease: a doseresponse meta-analysis of prospective cohort studies. European Journal of Epidemiology, 29(9), 613-619. doi:10.1007/s10654-014-9921-4

Kanthasamy, A. G., Kitazawa, M., Kanthasamy, A., \& Anantharam, V. (2005). Dieldrin-induced neurotoxicity: Relevance to Parkinson's disease pathogenesis. Neurotoxicology, 26(4), 701-719. doi:10.1016/j.neuro.2004.07.010

Kasabova-Angelova, A., Kondeva-Burdina, M., Mitkov, J., Georgieva, M., Tzankova, V., \& Zlatkov, A. (2020). Neuroprotective and MAOB inhibitory effects of a series of caffeine-8-thioglycolic acid amides. Brazilian Journal of Pharmaceutical Sciences, 56. doi:10.1590/s2175-97902019000318255

Kim, M., Jung, J., Jeong, N. Y., \& Chung, H. J. (2019). The natural plant flavonoid apigenin is a strong antioxidant that effectively delays peripheral neurodegenerative processes. Anatomical Science International, 94(4), 285-294. doi:10.1007/s12565-019-00486-2

Kim, S., Kwon, S. H., Kam, T. I., Panicker, N., Karuppagounder, S. S., Lee, S., . . Ko, H. S. (2019). Transneuronal Propagation of Pathologic alpha-Synuclein from the Gut to the Brain Models Parkinson's Disease. Neuron, 103(4), 627-+. doi:10.1016/j.neuron.2019.05.035

Kistner, A., \& Krack, P. (2014). Parkinson's disease: no milk today? Frontiers in Neurology, 5. doi:10.3389/fneur.2014.00172 
Kondeva-Burdina, M., Georgieva, M., Kasabova-Angelova, A., Tzankova, V., \& Zlatkov, A. (2019). Preliminary in vitro evaluation of neuroprotective and monoamine oxidase type B inhibitory effects of newly synthesized 8-aminocaffeines. Neural Regeneration Research, 14(6), 971-972. doi:10.4103/1673-5374.250573

Kuder, K. J., Zaluski, M., Schabikowski, J., Latacz, G., Olejarz-Maciej, A., Jasko, P., . . Kiec-Kononowicz, K. (2020). Novel, Dual Target-Directed Annelated Xanthine Derivatives Acting on Adenosine Receptors and Monoamine Oxidase B. Chemmedchem, 15(9), 772-786. doi:10.1002/cmdc.201900717

Liu, S. Y., Chan, P., \& Stoessl, A. J. (2017). The underlying mechanism of prodromal PD: insights from the parasympathetic nervous system and the olfactory system. Translational Neurodegeneration, 6, 9. doi:10.1186/s40035-017-0074-8

Ma, C. R., Molsberry, S., Li, Y. P., Schwarzschild, M., Ascherio, A., \& Gao, X. (2020). Dietary nicotine intake and risk of Parkinson disease: a prospective study. American Journal of Clinical Nutrition, 112(4), 1080-1087. doi:10.1093/ajcn/nqaa186

Manalo, R. V. M., \& Medina, P. M. B. (2018). Caffeine Protects Dopaminergic Neurons From Dopamine-Induced Neurodegeneration via Synergistic Adenosine-Dopamine D2-Like Receptor Interactions in Transgenic Caenorhabditis elegans. Frontiers in Neuroscience, 12. doi:10.3389/fnins.2018.00137

McDaniel, P. A., Solomon, G., \& Malone, R. E. (2005). The tobacco industry and pesticide regulations: Case studies from tobacco industry archives. Environmental Health Perspectives, 113(12), 1659-1665. doi:10.1289/ehp.7452

Miyazaki, I., Isooka, N., Kikuoka, R., Wada, K., Kitamura, Y., \& Asanuma, M. (2018). Neuroprotective effects of coffee ingredients against rotenone-induced neurodegeneration in parkinsonian model. Movement Disorders, 33, S85-S85. Retrieved from < Go to ISI>://WOS:000446176700184

Munoz, D. G., \& Fujioka, S. (2018). Caffeine and Parkinson disease: A possible diagnostic and pathogenic breakthrough. Neurology, 90(5), 205-206. doi:10.1212/wnl.0000000000004898

Nataf, S., Guillen, M., \& Pays, L. (2019). Common Neurodegeneration-Associated Proteins Are Physiologically Expressed by Human B Lymphocytes and Are Interconnected via the Inflammation/Autophagy-Related Proteins TRAF6 and SQSTM1. Frontiers in Immunology, 10, 16. doi:10.3389/fimmu.2019.02704

Nicholatos, J. W., Francisco, A. B., Bender, C. A., Yeh, T., Lugay, F. J., Salazar, J. E., . . Libert, S. (2018).

Nicotine promotes neuron survival and partially protects from Parkinson's disease by suppressing SIRT6. Acta Neuropathologica Communications, 6, 18. doi:10.1186/s40478-018-0625-y

Nielsen, S. S., Franklin, G. M., Longstreth, W. T., Swanson, P. D., \& Checkoway, H. (2013). Nicotine from Edible Solanaceae and Risk of Parkinson Disease. Annals of Neurology, 74(3), 472-477. doi:10.1002/ana.23884

Oertel, W., Muller, H., Schade-Brittinger, C., Kamp, C., Balthasar, K., Articus, K., . . Boyd, J. (2018). The NICPD-study - A randomized, placebo-controlled, double-blind, multi-centre trial to assess the disease- 
modifying potential of transdermal nicotine in early Parkinson's disease in Germany and N. America. Movement Disorders, 33, S159-S159. Retrieved from < Go to ISI>://WOS:000446176700345

Pervin, M., Unno, K., Ohishi, T., Tanabe, H., Miyoshi, N., \& Nakamura, Y. (2018). Beneficial Effects of Green Tea Catechins on Neurodegenerative Diseases. Molecules, 23(6). doi:10.3390/molecules23061297

Quik, M., O'Leary, K., \& Tanner, C. M. (2008). Nicotine and Parkinson's disease: Implications for therapy. Movement Disorders, 23(12), 1641-1652. doi:10.1002/mds.21900

Ribeiro, J. A., \& Sebastiao, A. M. (2010). Caffeine and Adenosine. Journal of Alzheimers Disease, 20, S3-S15. doi:10.3233/jad-2010-1379

Sampson, T. R., Debelius, J. W., Thron, T., Janssen, S., Shastri, G. G., Ilhan, Z. E., . . Mazmanian, S. K. (2016). Gut Microbiota Regulate Motor Deficits and Neuroinflammation in a Model of Parkinson's Disease. Cell, 167(6), 1469-+. doi:10.1016/j.cell.2016.11.018

Schlesinger, I., \& Schlesinger, N. (2008). Uric acid in Parkinson's disease. Movement Disorders, 23(12), $1653-1657$. doi: $10.1002 / \mathrm{mds} .22139$

Seidl, S., Santiago, J., Bilyk, H., \& Potashkin, J. (2014). The emerging role of nutrition in Parkinson's disease. Frontiers in Aging Neuroscience, 6(36). doi:10.3389/fnagi.2014.00036

Stefanis, L. (2012). alpha-Synuclein in Parkinson's Disease. Cold Spring Harbor Perspectives in Medicine, 2 (2), 23. doi:10.1101/cshperspect.a009399

Svensson, E., Horvath-Puho, E., Thomsen, R. W., Djurhuus, J. C., Pedersen, L., Borghammer, P., \& Sorensen, H. T. (2015). Vagotomy and Subsequent Risk of Parkinson's Disease. Annals of Neurology, 78(4), 522-529. doi:10.1002/ana.24448

Taura, J., Nolen, E. G., Cabre, G., Hernando, J., Squarcialupi, L., Lopez-Cano, M., . . Ciruela, F. (2018). Remote control of movement disorders using a photoactive adenosine A(2A) receptor antagonist. Journal of Controlled Release, 283, 135-142. doi:10.1016/j.jconrel.2018.05.033

Tseng, H. C., Wang, M. H., Chang, K. C., Soung, H. S., Fang, C. H., Lin, Y. W., . . Tsai, C. C. (2020). Protective Effect of (-)Epigallocatechin-3-gallate on Rotenone-Induced Parkinsonism-like Symptoms in Rats. Neurotoxicity Research, 37(3), 669-682. doi:10.1007/s12640-019-00143-6

Weindel, C. G., Bell, S. L., Vail, K. J., West, K. O., Patrick, K. L., \& Watson, R. O. (2020). LRRK2 maintains mitochondrial homeostasis and regulates innate immune responses to Mycobacterium tuberculosis. Elife, 9, 31. doi:10.7554/eLife.51071

Xu, Y., Xie, M. M., Xue, J. S., Xiang, L., Li, Y. L., Xiao, J., . . Wang, H. L. (2020). EGCG ameliorates neuronal and behavioral defects by remodeling gut microbiota and TotM expression in Drosophila models of Parkinson's disease. Faseb Journal, 34(4), 5931-5950. doi:10.1096/fj.201903125RR

Yan, R., Zhang, J., Park, H. J., Park, E. S., Oh, S., Zheng, H. Y., . . Mouradian, M. M. (2018). Synergistic neuroprotection by coffee components eicosanoyl-5-hydroxytryptamide and caffeine in models of 
Parkinson's disease and DLB. Proceedings of the National Academy of Sciences of the United States of America, 115(51), E12053-E12062. doi:10.1073/pnas.1813365115

Zhen, C., Li, D. M., Wang, H. Y., Wang, P., Zhang, W. J., Yu, J. T., . . Wang, X. (2019). Tea consumption and risk of Parkinson's disease: A meta-analysis. Neurology Asia, 24(1), 31-40. Retrieved from $<$ Go to ISI>://WOS:000463141900006

Zhou, W., Chen, L., Hu, X. Q., Cao, S. S., \& Yang, J. X. (2019). Effects and mechanism of epigallocatechin-3gallate on apoptosis and mTOR/AKT/GSK-3 beta pathway in substantia nigra neurons in Parkinson rats. Neuroreport, 30(2), 60-65. doi:10.1097/wnr.0000000000001149 